\title{
Enhancement of piezoelectric properties of lithium niobate thin films by different annealing parameters
}

\author{
Roman N. Zhukov ${ }^{1}$, K.S. Kushnerev ${ }^{1}$, Dmitry A. Kiselev ${ }^{1}$, Tatiana S. Ilina ${ }^{1}$, Ilya V. Kubasov ${ }^{1}$, \\ Alexander M. Kislyuk ${ }^{1}$, Mikhail D. Malinkovich ${ }^{1}$, Yuri N. Parkhomenko ${ }^{1}$ \\ 1 National University of Science and Technology MISiS, 4 Leninsky Prospekt, Moscow 119049, Russia \\ Corresponding author: Mikhail D. Malinkovich (malinkovich@yandex.ru)
}

Received 14 May 2020 • Accepted 25 June 2020 • Published 30 June 2020

Citation: Zhukov RN, Kushnerev KS, Kiselev DA, Ilina TS, Kubasov IV, Kislyuk AM, Malinkovich MD, Parkhomenko YuN (2020) Enhancement of piezoelectric properties of lithium niobate thin films by different annealing parameters. Modern Electronic Materials 6(2): 47-52. https://doi.org/10.3897/j.moem.6.2.54295

\begin{abstract}
Piezoelectric materials with useful properties find a wide range of applications including opto- and acousto- electronics. Lithium niobate in the form of a thin film is one of those promising materials and has a potential to improve ferroelectric random access memories devices, optical waveguides or acoustic delay lines by virtue of its physical characteristics, e.g. electro-optic coefficient, acoustic velocity, refractive indices etc. The key challenge to overcome is lithium nonstoichiometry as it leads to the appearance of parasite phases and thus aggravates physical and structural properties of a film. According to literature data, in order to obtain microcrystalline piezoelectric phase in previously amorphous films a set of methods is used. In our case we tried to synthesize LN films using congruent target and non-heated silicon substrate and then attain the piezoelectric phase by different annealing parameters. Afterwards LN films were compared to the ones synthesized on the silicon substrate with an additional buffer layer of platinum. Samples were studied by scanning probe microscope. Self-polarization vectors were defined. Based on domain structure images, the histograms of distribution of piezoresponse signals were built.
\end{abstract}

\section{Keywords}

Lithium niobate, rf magnetron sputtering, post-growth heat treatment, scanning probe microscopy, piezoelectric force microscopy, histogram of distribution of vertical and lateral piezoresponse

\section{Introduction}

Piezoelectrics are promising materials for various applications in actual and potential electronics, in particular opto- and acousto- electronics and guided photonics. One of the most substantial and interesting piezoelectric materials is lithium niobate $\left(\mathrm{LiNbO}_{3}\right)[1,2]$. This compound comprises unique and exceptional properties eligible to be implemented into diverse devices. Along with thermally stable piezoelectric modules and pyroelectric coefficients [1] this material also possesses unique opti- cal properties, which have a potential to upgrade optical waveguides, ferroelectric random access memories, high dielectric constant capacitors, acoustic delay lines, microwave tunable devices. Among those properties are electromechanical coupling coefficient $\left(\mathrm{K}^{2}\right)$ [3], electro-optic coefficient, acoustic velocity and refractive indices; thus, it is widely used in many diverse devices such as next-generation of high-frequency and/or wide-band RF filters or tuneable frequency filters adapted to the fifth generation of networks [4]. However physical and structural properties of LN are highly dependent on the lithium nonstoichiometry $[5,6]$. For instance, deficiency of lithium 
atoms substantially affects Curie temperature [7], elastic constants [8], density [9, 10], refractive indices [11], birefringence and many other characteristics. Therefore, the composition of the films has to be precisely controlled for large-scale devices production. Lithium is a light element and (after the growth of the film) lack of its atoms in LN compound leads to emergence of parasite non-piezoelectric phases (such as $\mathrm{LiNb}_{3} \mathrm{O}_{8}$ ) [12]. A number of steps were made to prevent this process, such as, heating the substrate or using of stoichiometric target or even target with lithium excess. Another way to improve the quality of the film is to use a substrate with metal buffer layers. In our case we used congruent target and tried to obtain films on a non-heated silicon substrate to minimize lithium volatility and then enhance film's piezoelectric properties and crystallinity by post-growth heat treatment. Therefore, the goal of this work was to find optimal annealing parameters of an initially amorphous LN thin film (grown on a silicon substrate without any additional buffer layers) in order to obtain microcrystalline piezoelectric phase.

\section{Experimental}

For the experimental research the thin film of LN was grown by RF magnetron sputtering. The deposition of the thin film was performed on a silicon substrate of (111) orientation in atmosphere of argon and oxygen with ratio of $1: 1$. The substrate was preliminarily cleaned with argon gun for 5 minutes before the beginning of deposition process.

All samples were produced by magnetron sputtering in SUNPLA 40TM vacuum chamber (South Korea) [13]. The magnetron power equaled $150 \mathrm{~W}$ with the pressure in vacuum chamber where deposition process was carried out being $0.5 \mathrm{~Pa}$. The film thickness equaled 300 $\mathrm{nm}$. Once the growth process was accomplished, initially amorphous films were annealed in order to improve the crystallinity as well as to increase the share of piezoelectric phase of (R3c) $\mathrm{LiNbO}_{3}$ in the film. Post-growth heat treatment parameters were different for each sample.

The annealing was performed in the muffle furnace at the temperature of $700^{\circ} \mathrm{C}$, the optimal annealing temperature: it lowers thin film's surface roughness and contributes to the highest degree to the structuring of the ferroelectric phase [13]. Samples 1 and 2 were both heated to $700{ }^{\circ} \mathrm{C}$ for 12 minutes, afterwards Sample 1 was held at this temperature for 1 more minute while Sample 2 - for 5 minutes.

Samples 3 and 4 were heated to $700{ }^{\circ} \mathrm{C}$ quicker - for 5 minutes. Once the process was completed Sample 3 was removed from the furnace and Sample 4 was held at that temperature for one more minute before it was taken out from the furnace.

Samples 5 and 6 were heated to $700{ }^{\circ} \mathrm{C}$ for 2 minutes. In this case Sample 5 was right afterwards removed from the furnace while Sample 6 was held there for 1 minute at the same temperature of $700{ }^{\circ} \mathrm{C}$.

All six samples were cooled naturally in the atmosphere.
Studies of topography, domain structure and residual hysteresis loops were carried out on SEM with the help of MFP-3D SA scanning probe microscope (Asylum Research, USA) using Asyelec-02 cantilever (Asylum Research, USA). The subsequent analysis of topography images with WxSM and Gwyddion programs allowed to determine roughness and grain size of the examined thin films. Visualization of domain structure was realized by piezoelectric force microscopy.

Domain structure images were obtained by applying AC voltage of $5 \mathrm{~V}$, frequency of $142 \mathrm{kHz}$ by phase shift of $270^{\circ}$ to cantilever.

With the help of scanning probe microscopy, it is possible to define the direction of self-polarization $\mathrm{P}_{\mathrm{s}}$ in examined materials. For this purpose, the histograms of the distribution of piezoresponse signals based on domain structure images were built.

\section{Results and discussion}

In order to determine the direction of polarization $\mathrm{P}_{\mathrm{s}}$, once a piezoresponse magnitude image is obtained, a histogram was generated and the peak values were noted. Moreover, the distribution of piezoresponse signals allows to make a conclusion on the volume of piezoelectric phase in thin films samples.

First pair of samples (1 and 2) was slowly heated up to $700{ }^{\circ} \mathrm{C}$ for 12 minutes with holding time of 1 and 5 minutes respectively. The temperature of $700{ }^{\circ} \mathrm{C}$ was chosen with regards to the work of D. A. Kiselev, R. N. Zhukov et al [13], where a considerable share of non-piezoelectric LiN$\mathrm{b}_{3} \mathrm{O}_{8}$ phase was detected at higher $\left(1000{ }^{\circ} \mathrm{C}\right)$ temperature while annealing at lower temperature $\left(550{ }^{\circ} \mathrm{C}\right)$ led to decrease in film crystallinity. The corresponding diffractogram is presented at Figure 3. As the histograms show (Figure 1a, b) there are several not intensive peaks different from zero. They indicate a small part of domains with multidirectional self-polarization vectors. Nevertheless, the intensity of zero-peak demonstrates the presence of a non-piezoelectric phase of high volume in LN films. The intensity of piezoresponse of both samples in lateral and vertical direction was almost the same with a maximum of 5,0E8 arbitrary units.

In order to decrease the effect of lithium volatility during the annealing process it was suggested to reduce both heating and holding times for Samples 3 and 4. As the histograms show (Figure 1c, d), both samples' zero peaks in vertical direction moved slightly away from zero, while these peaks were not as narrow as for Samples 1 and 2 . This is a good sign of possible presence of piezoelectric phase. However, self-polarization vector forwarded from the interface has an extremely low value.

Samples 5 and 6 were heated to $700^{\circ} \mathrm{C}$ even quicker (in 2 minutes) but their histograms (Figure $2 \mathrm{e}, \mathrm{f}$ ) reveal an almost complete absence of piezoelectric phase in LN films.

Possibly the films could not crystallize for such a short time and a part of the samples was still amorphous or far enough from microcrystalline structure. 

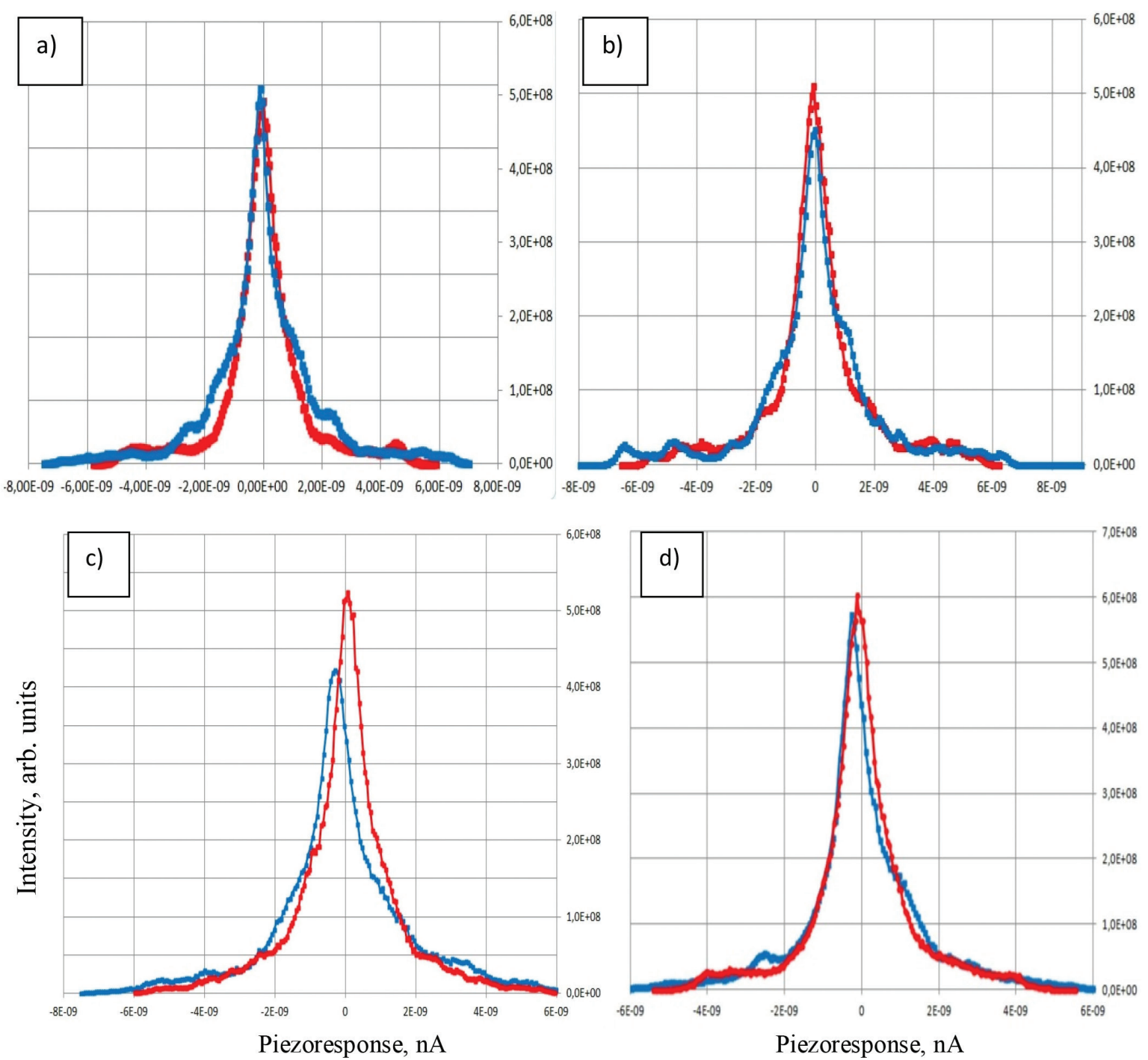

Figure 1. Histograms of distribution of piezoresponse signals for Samples 1-4 (a,b, c, d respectively). Blue line is the vertical piezoresponse, red line is the lateral piezoresponse.

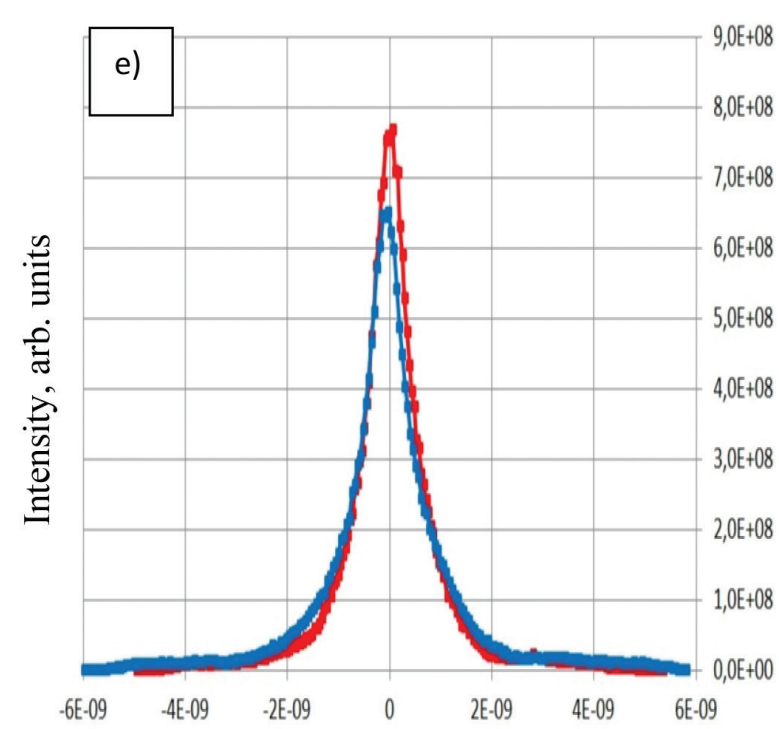

Piezoresponse, $\mathrm{nA}$

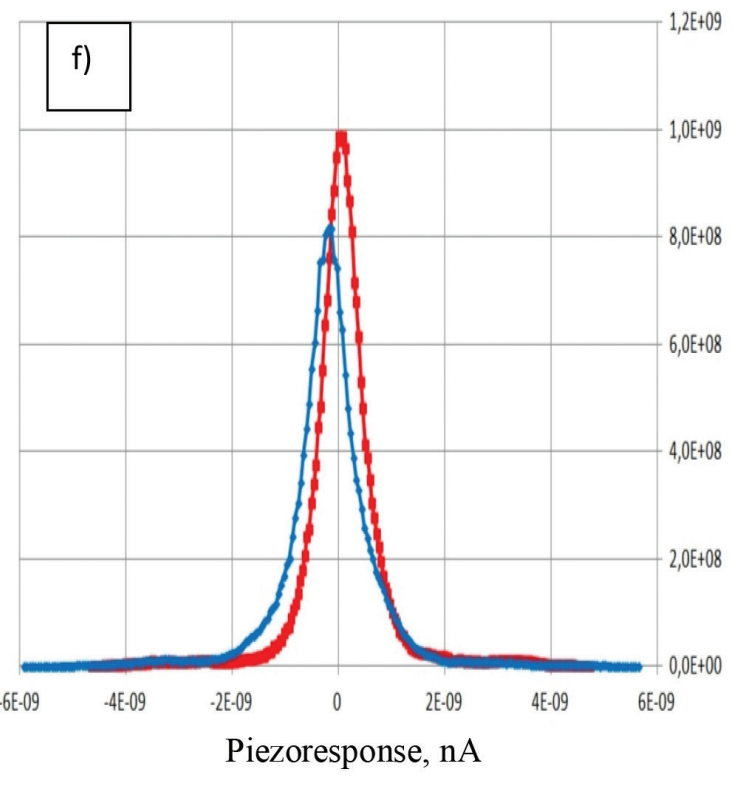

Piezoresponse, nA

Figure 2. Histograms of distribution of piezoresponse signals for Samples 5-6 (e,f respectively). Blue line is the vertical piezoresponse, red line is the lateral piezoresponse. 


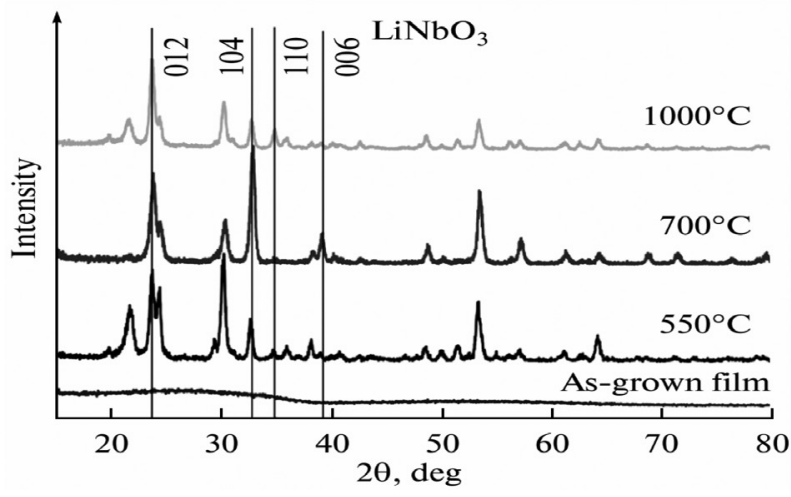

Figure 3. X-ray diffraction patterns of an as-grown $\mathrm{LiNbO}_{3}$ film and films heat-treated at different temperatures from the work of Kiselev et al. [13].
The roughness of all samples varied between 1.4$2.4 \mathrm{~nm}$. Figure 4 displays the topography and domain structures of Samples 1-4, while Figure 5 shows the same for Samples 5 and 6.

The combined results of heating and holding times, roughness and intensity can be observed from the Table 1 .

According to all the histograms presented earlier in the paper (Figures 1 and 2), we can conclude that it is extremely challenging to obtain successful lithium niobate films with satisfying piezoelectric characteristics on "clean" silicon substrates. The probable reason for this is that there is always a layer of silicon oxide $\mathrm{SiO}_{x}$ on the surface of silicon unless it is purposely removed before lithium niobate is deposited. Silicon oxide is an effective getter for $\mathrm{Li}_{2} \mathrm{O}$ [14], which promotes lithium niobate film
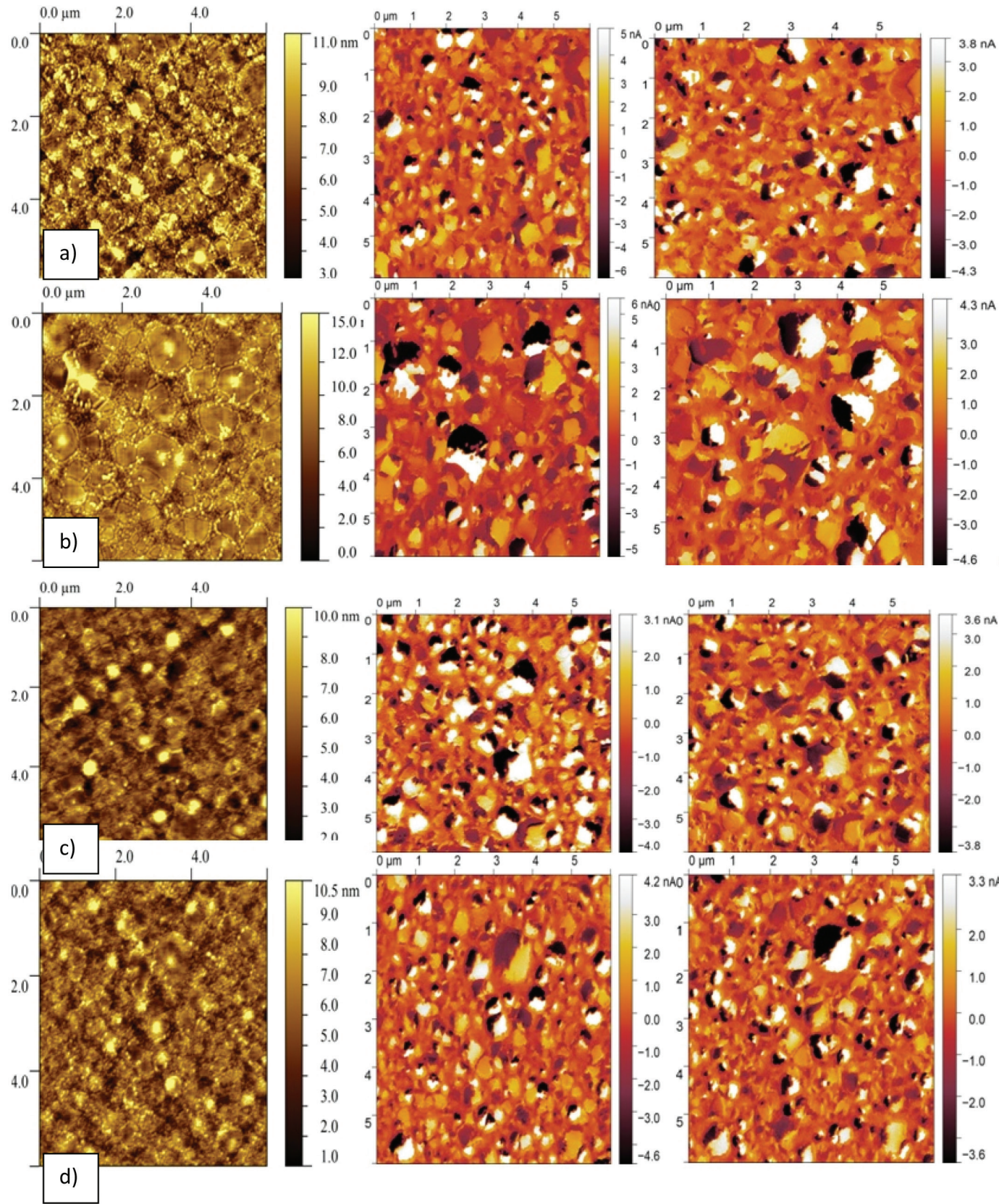

Figure 4. Topography and domain structures of samples 1-4 (a, b, c, d). From left to right: topography, VPFM, LPFM. 

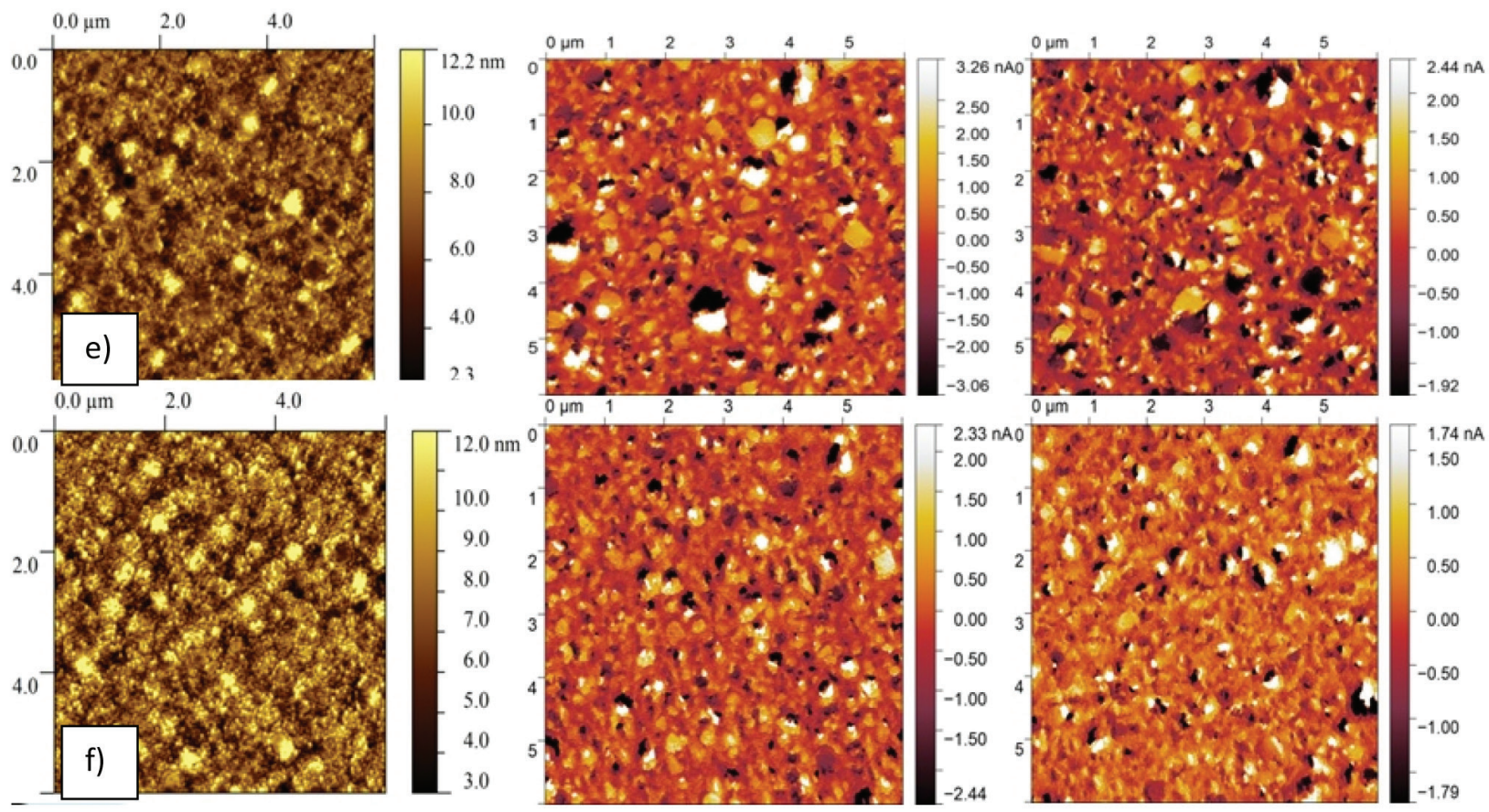

Figure 5. Topography and domain structures of Samples 5 and 6 (e and f). From left to right: topography, VPFM, LPFM.

Table 1. The combined results of annealing parameters and following roughness and intensity examination.

\begin{tabular}{lcccc}
\hline Sample & $\begin{array}{c}\text { Heating time, } \\
\text { minutes (up } \\
\left.\text { to } \mathbf{7 0 0}^{\circ} \mathbf{C}\right)\end{array}$ & $\begin{array}{c}\text { Holding time, } \\
\text { minutes (at } \\
\left.\mathbf{7 0 0}^{\circ} \mathbf{C}\right)\end{array}$ & $\begin{array}{c}\text { Roughness, } \\
\mathbf{n m}\end{array}$ & $\begin{array}{c}\text { Intensity, } \\
\text { arb. units }\end{array}$ \\
\hline 1 (a) & 12 & 1 & 1.88 & $5.0 \mathrm{E} 8$ \\
2 (b) & 12 & 5 & 2.35 & $5.2 \mathrm{E} 8$ \\
3 (c) & 5 & 0 & 1.42 & $5.5 \mathrm{E} 8$ \\
4 (d) & 5 & 1 & 1.54 & $6.0 \mathrm{E} 8$ \\
5 (e) & 2 & 0 & 2.00 & $7.8 \mathrm{E} 8$ \\
6 (f) & 2 & 1 & 2.22 & $1.0 \mathrm{E} 9$ \\
\hline
\end{tabular}

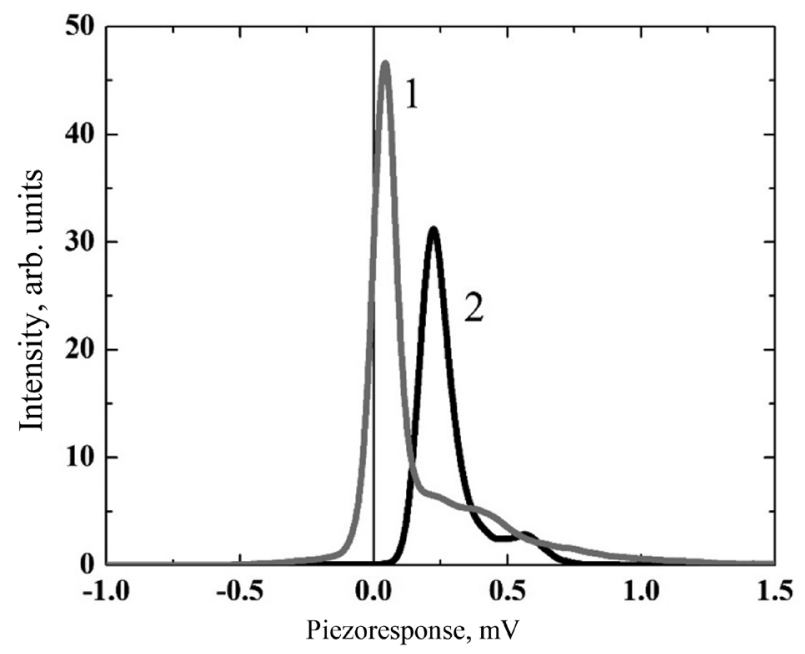

Figure 6. Histograms of distribution of piezoresponse signals for $\mathrm{LiNbO}_{3} / \mathrm{SiO}_{x} / \mathrm{Si}$ (line 1) and $\mathrm{LiNbO}_{3} / \mathrm{Pt} / \mathrm{Si}$ (line 2) heterostructures [14].

depletion by lithium. Thus, it can be expected that more stoichiometric compound of a lithium niobate film on the surface of silicon can be obtained by generating an anti-diffusion barrier between lithium niobate and the substrate. For example, a thin layer of platinum can be used as an anti-diffusion barrier preventing the loss of lithium from the film [15]. Figure 6 shows the distribution histogram of piezoresponse signal received on a lithium niobate film containing a platinum sublayer [16], compared with the result obtained on a film without a platinum sublayer. It can be observed that piezoresponse and, therefore, the part of the piezoelectric phase on films with a platinum sublayer is much larger while the nonpiezoelectric phase is practically absent which indicates sufficiently high quality of such material.

\section{Conclusion}

By changing annealing parameters (heating and holding times) we tried to enhance piezoelectric properties of lithium niobate thin films grown on a "cold" Si (111) substrate by RF magnetron sputtering. According to piezoresponse signal distribution there is a small volume of differently oriented domains (which embody the existence of piezoelectric phase) in samples heated up to $700{ }^{\circ} \mathrm{C}$ for 12 minutes (with holding times of 1 and 5 minutes) and in samples heated for 5 minutes (with holding times of 0 and 1 minute). The roughness of those films is extremely low and lies between 1.4 and $2.4 \mathrm{~nm}$.

Nevertheless, generally we can reveal that samples contain an extremely small amount of piezoelectric component which might be the consequence of poorly selected annealing parameters. Presumably at shortening heating time LN film is unable to form a stable multi-domain structure and 
predominant formation of isolated single grains happens. Though it would be tremendously beneficial and advantageous to obtain lithium niobate films on silicon substrates without any additional layers, the task seems immensely challenging. We conducted a series of annealing experiments and came to the conclusion that the most successful LN films were grown on the substrates with such buffer layers as platinum. Those films contained a larger amount of piezoelectric phase and showed better piezoresponse.

Further experiments on the selection of optimal annealing parameters are necessary for enhancement and increase of the piezoelectric phase in lithium niobate thin films.

\section{References}

1. Kuzminov Y.S. Electroopticheskiy i nelineynoopticheskiy kristall niobata litiya [Electrooptical and Nonlinear Optical Lithium Niobate Crystal]. Moscow: Nauka, 1987: 263 p. (In Russ.)

2. Volk T., Wohlecke M. Lithium Niobate: Defects, Photorefraction and Ferroelectric Switching, Springer Science \& Business Media, 2008: $250 \mathrm{p}$.

3. Yamada K., Takemura H., Inoue Y., Omi T., Matsumura S. Effect of $\mathrm{Li} / \mathrm{Nb}$ Ratio on the SAW Velocity of $128^{\circ} \mathrm{Y}-\mathrm{X} \mathrm{LiNbO}_{3}$ Wafers. Japanese Journal of Applied Physics. 1987; 26-2: 219-222. https://doi.org/10.7567/JJAPS.26S2.219

4. Bartasyte A., Margueron S., Baron T., Oliveri S., Boulet P. Toward High-Quality Epitaxial $\mathrm{LiNbO}_{3}$ and $\mathrm{LiTaO}_{3}$ Thin Films for Acoustic and Optical Applications. Advanced Materials Interfaces. 2017; 4 (8): 1600998 (36 pp.). https://doi.org/10.1002/admi.201600998

5. Bordui P.F., Norwood R.G., Bird C.D., Calvert G.D. Compositional uniformity in growth and poling of large-diameter lithium niobate crystals. Journal of Crystal Growth. 1991; 113(1-2): 61-68. DOI: 10.1016/0022-0248(91)90009-T

6. Bordui P.F., Norwood R.G., Bird C.D., Carella J.T. Stoichiometry issues in single-crystal lithium tantalate. Journal of Applied Physics. 1995; 78(7): 4647-4650. https://doi.org/10.1063/1.359811

7. Tian L., Gopalan V., Galambos L. Domain reversal in stoichiometric $\mathrm{LiTaO}_{3}$ prepared by vapor transport equilibration. $A p$ plied Physics Letters. 2004; 85(19): 4445-4447. https://doi org/10.1063/1.1814436

8. Kushibiki J., Takanaga I., Komatsuzaki S., Ujiie T. Chemical composition dependences of the acoustical physical constants of LiN$\mathrm{bO}_{3}$ and $\mathrm{LiTaO}_{3}$ single crystals. Journal of Applied Physics. 2002; 91(10): 6341-6349. https://doi.org/10.1063/1.1467608

9. Iyi N., Kitamura K., Izumi F., Yamamoto J.K., Hayashi T., Asano H., Kimura S. Comparative study of defect structures in lithium niobate with different compositions. Journal of Solid State Chemistry. 1992; 101(2): 340-352. https://doi.org/10.1016/0022 4596(92)90189-3

\section{Acknowledgements}

The work was supported by the Ministry of Science and Higher Education of the Russian Federation as a part of the State Assignment (basic research, Project No. 07182020-0031 "New magnetoelectric composite materials based on oxide ferroelectrics having an ordered domain structure: production and properties"). The studies were performed on the equipment of Center for Shared Use "Materials Science and Metallurgy" at the National University of Science and Technology "MISiS".
10. Kushibiki J., Ohashi Y., Hirohashi J. Ultrasonic microspectroscopy of congruent $\mathrm{LiNbO}_{3}$ crystals. Journal of Applied Physics. 2005; 98(12): 123507 (1-8). https://doi.org/10.1063/1.2146076.

11. Schlarb U., Betzler K. Refractive indices of lithium niobate as a function of wavelength and composition. Journal of Applied Physics. 1993; 73(7): 3472-3476. https://doi. org/10.1063/1.352951

12. Prezas P. R. S, Graça M. P. F Structural Characterization of Lithium Niobate Nanoparticles Prepared by the Sol-Gel Process, Using X-Ray and Raman Spectroscopy and Scanning Electron Microscopy. Applications of Molecular Spectroscopy to Current Research in the Chemical and Biological Sciences, 2016. http://dx.doi. org/10.5772/64395

13. Kiselev D.A., Zhukov R.N., Bykov A.S., Voronova M.I., Shcherbachev K.D., Malinkovich M.D., Parkhomenko Y.N. Effect of annealing on the structure and phase composition of thin electro-optical lithium niobate films. Inorganic Materials, 2014; 50(4): 419-422. https://doi.org/10.1134/s0020168514040074

14. Yamamoto K., Mizuuchi K., Takeshige K., Sasai Y., Taniuchi T. Characteristics of periodically domain-inverted $\mathrm{LiNbO3}$ and $\mathrm{LiTaO} 3$ waveguides for second harmonic generation. Journal of Applied Physics. 1991; 70(4): 1947-1951. https://doi. org $/ 10.1063 / 1.349477$

15. Ohlendorf G., Richter D., Sauerwald J., Fritze H. High-temperature electrical conductivity and electro-mechanical properties of stoichiometric lithium niobate. Diffusion Fundamentals. 2008; 8: 6.1-6.7. URL: https://nbn-resolving.org/urn:nbn:de:bsz:15-qucosa-192902

16. Zhukov R.N., Kiselev D.A., Ilina T.S., Temirov A.A., Kubasov I.V., Bykov A.S., Savchenko A.G. Effect of a Platinum Sublayer on the Microstructure and Spontaneous Polarization of Thin Lithium Niobate Films Deposited on a Silicon Substrate. Bulletin of the Russian Academy of Sciences: Physics. 2018; 82(9): 1232-1235. https://doi.org/10.3103/s1062873818090265 